\title{
Efeito de soluções eletrolíticas enterais com diferentes osmolaridades sobre o perfil eletrolítico e bioquímico de equinos ${ }^{1}$
}

\author{
José D. Ribeiro Filho ${ }^{2 *}$, Sheila K. Farias ${ }^{3}$, Athina C. Donner ${ }^{3}$, Dyego P. Oliveira ${ }^{3}$, José \\ D. Guimarães ${ }^{2}$, Maria V. Souza², Cláudio L.N. Gomes ${ }^{4}$ e Marcel F.B. Avanza ${ }^{3}$
}

\begin{abstract}
Ribeiro Filho J.D., Farias S.K., Donner A.C., Oliveira D.P., Guimarães J.D., Souza M.V., Gomes C.L.N. \& Avanza M.F.B. 2014. [Horses treated with enteral electrolyte solutions with differents osmolarities.] Efeito de soluções eletrolíticas enterais com diferentes osmolaridades sobre o perfil eletrolítico e bioquímico de equinos. Pesquisa Veterinária Brasileira 34(2):179-184. Departamento de Veterinária, Universidade Federal de Viçosa, Avenida P.H. Rolfs s/n, Viçosa, MG 36570-000, Brazil. E-mail: dantas@ufv.br

We compared the effects of electrolyte solutions with different osmolarities administered through enteral route by naso-esophageal probe of small-caliber with continuos flow on the electrolytic and biochemical profile in horses. Six adult females were used in two simultaneous $6 \times 3$ Latin squares mixed model. The animals were divided into three groups and received the following treatments: HipoMalt - $5 \mathrm{~g}$ of sodium chloride, $0.5 \mathrm{~g}$ of potassium chloride, $0.2 \mathrm{~g}$ of magnesium pidolate, $1 \mathrm{~g}$ of calcium gluconate and $10 \mathrm{~g}$ of maltodextrin diluted in $1.000 \mathrm{~mL}$ of water $\left(181 \mathrm{mOsmol} \mathrm{L}^{-1}\right)$; HipoDext $-5 \mathrm{~g}$ of sodium chloride, $0.5 \mathrm{~g}$ of potassium chloride, $0.2 \mathrm{~g}$ of magnesium pidolate, $1 \mathrm{~g}$ of calcium gluconate and $10 \mathrm{~g}$ of dextrose diluted in $1.000 \mathrm{~mL}$ of water $\left(228 \mathrm{mOsmol} \mathrm{L}^{-1}\right)$; IsoProp - $5 \mathrm{~g}$ of sodium chloride, $0.5 \mathrm{~g}$ of potassium chloride, $0.2 \mathrm{~g}$ of magnesium pidolate, $1 \mathrm{~g}$ of calcium gluconate and $10 \mathrm{~g}$ of calcium propionate diluted in $1.000 \mathrm{~mL}$ of water $\left(282 \mathrm{mOsm} \mathrm{L}^{-1}\right)$. The hypotonic electrolyte solutions containing dextrose (HipoDext) and maltodextrin (HipoMalt) were more effective in increase the rate glucose without causing electrolyte imbalance. Treatment with calcium propionate (IsoProp) besides increasing plasma lactate had no effect on blood glucose.
\end{abstract}

INDEX TERMS: Enteral fluid therapy, hypotonic, isotonic, plasmatic volume, electrolytes.

RESUMO.- Foram comparados os efeitos de soluções eletrolíticas com diferentes osmolaridades administradas via enteral por sonda nasoesofágica de pequeno calibre, em fluxo contínuo, sobre o perfil bioquímico em equinos. Foram utilizadas seis fêmeas adultas em dois quadrados latinos 6x3 simultâneos em modelo misto. Os animais foram distribuídos em três grupos e cada grupo submetido aos

\footnotetext{
${ }^{1}$ Recebido em 19 de setembro de 2013.

Aceito para publicação em 23 de fevereiro de 2014.

${ }^{2}$ Departamento de Veterinária, Universidade Federal de Viçosa (UFV), Avenida P.H. Rolfs s/n, Viçosa, MG 36570-000, Brasil. *Autor para correspondência: dantas@ufv.br

${ }^{3}$ Pós-Graduando em Medicina Veterinária, UFV, Avenida P.H. Rolfs s/n, Viçosa, MG 36570-000.

${ }^{4}$ Departamento de Clínicas Veterinárias, Universidade Estadual do Maranhão, Cidade Universitária Paulo VI, s/n, Tirirical, São Luís, MA 65055310, Brasil.
}

seguintes tratamentos: HipoMalt - $5 \mathrm{~g}$ de cloreto de sódio, $0,5 \mathrm{~g}$ de cloreto de potássio, $0,2 \mathrm{~g}$ de pidolato de magnésio, $1 \mathrm{~g}$ de gluconato de cálcio e $10 \mathrm{~g}$ de maltodextrina diluídos em $1.000 \mathrm{~mL}$ de água (181mOsmol L $\left.\mathrm{m}^{-1}\right)$, HipoDext - $5 \mathrm{~g}$ de cloreto de sódio, $0,5 \mathrm{~g}$ de cloreto de potássio, $0,2 \mathrm{~g}$ de pidolato de magnésio, $1 \mathrm{~g}$ de gluconato de cálcio e $10 \mathrm{~g}$ de dextrose diluídos em $1.000 \mathrm{~mL}$ de água (228mOsmol L ${ }^{-1}$ ) e IsoProp - $5 \mathrm{~g}$ de cloreto de sódio, 0,5g de cloreto de potássio, 0,2g de pidolato de magnésio e $10 \mathrm{~g}$ de propionato de cálcio diluídos em $1.000 \mathrm{~mL}$ de água $\left(282 \mathrm{mOsm} \mathrm{L}^{-1}\right)$. As soluções contendo dextrose (HipoDext) e maltodextrina (HipoMalt) foram mais eficazes em aumentar a taxa glicêmica sem ocasionar desequilíbrio eletrolítico. Já o tratamento com propionato de cálcio (IsoProp) além de aumentar o lactato plasmático não teve efeito sobre a glicemia.

TERMOS DE INDEXAÇÃO: Hidratação enteral, hipotônica, volume plasmático, eletrólito. 


\section{INTRODUÇÃO}

Uma solução eletrolítica eficiente para reidratar equinos tem que ter na sua composição água, eletrólitos e, também, uma fonte de energia, que deve ser administrada de acordo com as necessidades do paciente e no seu perfil bioquímico. Dessa forma, algumas vezes, torna-se necessário acrescentar, diminuir ou suprimir determinado elemento na composição da solução eletrolítica enteral para aumentar o seu valor terapêutico (Ribeiro Filho et al. 2011). Usualmente essa prática é utilizada nos pacientes que apresentam distúrbios gastrintestinais, como por exemplo na diarreia e cólica (Rainger \& Dart 2006).

Os constituintes de uma solução eletrolítica para hidratação enteral em equinos são o sódio, potássio, cloreto, cálcio, magnésio e uma fonte de energia, que pode ser a glicose, dextrose ou maltodextrina. Entretanto, no Brasil, a disponibilidade desses produtos para uso em equinos ainda são poucos. A maioria é recomendada para recomposição das perdas hidroeletrolíticas após o exercício físico, além disso, muitos deles não são oriundos de estudos experimentais controlados (Teixeira-Neto et al. 2004, Donner 2013).

As soluções eletrolíticas enterais foram originalmente desenvolvidas na medicina humana para o tratamento da diarreia associada à cólera (Smith 2009). Embora a terapia de reidratação oral moderna tenha sido introduzida na década de 50, o seu potencial não foi totalmente valorizado até o começo dos anos 70 , porque, a reposição eletrolítica, antes desse período a hidratação intravenosa era comumente utilizada, mesmo nas desidratações discretas e moderadas (McClure 2001). Em veterinária, especialmente na medicina equina, a hidratação enteral já ocupa espaço considerável e tem contribuído sobremaneira para redução da taxa de mortalidade, principalmente na síndrome cólica e na diarreia, apesar dos ensaios controlados ainda serem escassos (Avanza et al. 2009, Ribeiro Filho et al. 2012, Pessin et al. 2013).

Estudos experimentais em humanos e ratos tem demonstrado que soluções eletrolíticas orais hipotônicas proporcionam melhor absorção de água do que as isotônicas, sinalizando que as soluções de reidratação oral devem apresentar baixa osmolaridade para maximizar a absorção de água (Hunt et al. 1992). A osmolaridade ideal das soluções eletrolíticas enterais utilizadas em equinos ainda é desconhecida, isso ocorre porque os estudos experimentais sobre esse tema na medicina veterinária ainda são escassos. Até o momento, a maioria dos ensaios experimentais com hidratação enteral em equinos foram realizados com soluções eletrolíticas isotônicas (Lopes et al. 2002, Ribeiro Filho et al. 2007, Avanza et al. 2009, Farias et al. 2011, Gomes et al. 2012), buscando-se determinar a melhor composição dessas soluções. Além do mais, apesar dessa carência, a maior parte dos experimentos enfocando a tonicidade dos soros enterais não utilizou solução eletrolítica hipotônica, mas sim a água (Sosa León et al. 1995b, Marlin et al. 1998).

O presente estudo objetivou avaliar o efeito de soluções eletrolíticas contendo diferentes osmolaridades administradas por via enteral em fluxo contínuo durante 12 horas, baseado no perfil eletrolítico e bioquímico de equinos.

\section{MATERIAL E MÉTODOS}

O presente ensaio foi realizado na cidade de Viçosa-MG. Ela está localizada em uma altitude de aproximadamente 649 metros, com coordenadas geográficas o paralelo de $20^{\circ}, 45^{\prime}, 14$ ", latitude S, e meridiano de $42^{\circ}, 52^{\prime} 54^{\prime \prime}$, longitude W Gr. Durante o ensaio a temperatura média foi de $26^{\circ} \mathrm{C}$ e a umidade relativa do ar de $72 \%$.

Utilizaram-se seis equinos hígidos, fêmeas (não gestantes), sem raça definida, com idade média de dois anos, bom escore corporal (Speirs 1997) e peso médio de $258 \mathrm{~kg}$. Uma semana antes do experimento após avaliação clínica, foi feito o controle de ectoparasitas (deltametrina a 0,025\%) e endoparasitas (praziquantel + ivermectina). Os animais foram alojados em baias individuais para adaptação, onde receberam água e feno de coast cross (Cynodon dactylon) à vontade, ração comercial concentrada duas vezes ao dia, correspondendo a $1 \%$ do peso corporal, e suplemento mineral, $50 \mathrm{~g}$ ao dia.

Os equinos foram distribuídos aleatoriamente em três grupos, cada um contendo seis animais. Foi realizado cross over 6x3 (seis equinos $\mathrm{x}$ três tratamentos). 0 intervalo entre os períodos de tratamento foi de sete dias (Quadro 1). Os tratamentos foram assim constituídos: o grupo HipoDext foi tratado com solução eletrolítica contendo $5 \mathrm{~g}$ de cloreto de sódio, $0,5 \mathrm{~g}$ de cloreto de potássio, $0,2 \mathrm{~g}$ de pidolato de magnésio, $1 \mathrm{~g}$ de gluconato de cálcio e $10 \mathrm{~g}$ de dextrose diluídos em $1.000 \mathrm{~mL}$ de água (osmolaridade mensurada: $228 \mathrm{mMol} \mathrm{L}^{-1}$; pH: 6,22); o grupo HipoMalt recebeu solução eletrolítica contendo $5 \mathrm{~g}$ de cloreto de sódio, $0,5 \mathrm{~g}$ de cloreto de potássio, $0,2 \mathrm{~g}$ de pidolato de magnésio, $1 \mathrm{~g}$ de gluconato de cálcio e $10 \mathrm{~g}$ de maltodextrina diluídos em $1.000 \mathrm{~mL}$ de água (osmolaridade mensurada: $181 \mathrm{mMol} \mathrm{L}^{-1}$; $\mathrm{pH}$ : 6,25) e no grupo IsoProp foi administrada solução eletrolítica composta por $5 \mathrm{~g}$ de cloreto de sódio, $0,5 \mathrm{~g}$ de cloreto de potássio, $0,2 \mathrm{~g}$ de pidolato de magnésio e $10 \mathrm{~g}$ de propionato de cálcio diluídos em $1.000 \mathrm{~mL}$ de água (osmolaridade mensurada: $282 \mathrm{mMol} \mathrm{L}^{-1}$; pH: 7,71). As soluções eletrolíticas enterais foram administradas na dose de $15 \mathrm{~mL} \mathrm{~kg}^{1} \mathrm{~h}^{-1}$ durante 12 horas em fluxo contínuo por sonda nasoesofágica de pequeno calibre $(5,7 \mathrm{~mm}$ de diâmetro e $1,5 \mathrm{~m}$ de comprimento).

Para a realização das análises bioquímicas, o sangue foi coletado em frascos vacutainer com fluoreto de sódio para obtenção de plasma e, em frascos vacutainer siliconizados sem anticoagulante, para obtenção do soro. No plasma foram determinados lactato (colorimétrico enzimático) e glicose (colorimétrico enzimático), enquanto que no soro foram mensurados sódio (fotometria de chama), potássio (fotometria de chama), cálcio ionizado (íon seletivo), cloreto (colorimétrico enzimático), proteínas séricas totais (colorimétrico enzimático), magnésio total (colorimétrico enzimático), ureia (colorimétrico enzimático), creatinina (colorimétrico enzimático). A osmolaridade sérica foi mensurada por

Quadro 1. Distribuição dos equinos nos tratamentos

\begin{tabular}{lcc}
\hline Período & Animais & Tratamentos \\
\hline 1o período & 1 e 2 & SEDext \\
1o período & 3 e 4 & SEMalt \\
1o período & 5 e 6 & SEProp \\
& Sete dias de intervalo & \\
2o período & 5 e 6 & SEDext \\
2o período & 1 e 2 & SEMalt \\
2o período & 3 e 4 & SEProp \\
& Sete dias de intervalo & \\
3o período & 3 e 4 & SEDext \\
3o período & 5 e 6 & SEMalt \\
3o período & 1 e 2 & SEProp
\end{tabular}

$\overline{\text { SEDext }=\text { solução eletrolítica enteral }}$ contendo dextrose. SEMalt $=$ solução eletrolítica enteral contendo maltodextrina. SEProp = solução eletrolítica enteral contendo propionato de cálcio. 
osmômetro. Todos os testes foram realizados observando as mensurações propostas por Lopes et al. (2002) e Alves et al. (2005).

As coletas de sangue para realização das análises laboratoriais foram realizadas sempre no mesmo horário (07:00, 13:00 e 19:00) nos seguintes intervalos: T0 (imediatamente antes da hidratação), T6 (seis horas de hidratação), T12 (12 horas e término da hidratação) e T24 (doze horas após o término da hidratação).

As variáveis quantitativas foram submetidas aos testes de Normalidade (Lilliefors) e Homocedasticidade (Cochran). Para avaliação do efeito dos tratamentos, utilizou-se análise de variância baseada em planejamento de medidas repetidas, ou seja, foi avaliado cada tratamento em vários tempos. Foi analisada também a influência do tempo e a interação entre o tratamento e o tempo. Quando a análise foi significativa para um ou mais fatores, utilizou-se o teste de Tukey (SAS 2000). Todas as análises foram interpretadas considerando o nível de significância de 5\% de probabilidade de erro $(\mathrm{p}<0,05)$.

O delineamento experimental foi submetido ao comitê de ética do Departamento de Veterinária da Universidade Federal de Viçosa, sendo aprovado sob o protocolo de número 05/2010.

\section{RESULTADOS E DISCUSSÃO}

Em relação ao sódio, durante toda a fase experimental (T0 a T24) não foi observada diferença nos valores médios entre os tratamentos HipoMalt, IsoProp e HipoDext $(p>0,05)$. 0 mesmo ocorreu nos tratamentos ao longo do período de hidratação (T0 a T12). Porém, constatou-se decréscimo nos valores desse eletrólito nos tratamentos HipoMalt e IsoProp no T24 (Quadro 2), ressaltando-se que o mesmo foi discreto, a prova disso é que apesar da diminuição os valores mantiveram-se na faixa de normalidade (Kaneko et al. 2008). Além do mais, o seu aparecimento ocorreu 12 horas após o término da hidratação (T24), sinalizando que o volume e a composição das soluções eletrolíticas enterais administradas aos animais não tiveram influência sobre esse resultado, pois como citaram Lindger \& Acker (2013) o esvaziamento gástrico de soluções eletrolíticas e a absorção intestinal de eletrólitos contidos nelas são rápidos, e, como já tinha transcorrido 12 horas subsequentes ao término da hidratação, é provável que o aparecimento desse evento deveu-se a ingestão de água e alimento pelos animais no pós-tratamento (T12 a T24).

Quando se administra uma solução eletrolítica reidratante, espera-se que além de corrigir os desequilíbrios existentes ela não produza alterações indesejáveis na concentração sérica ou plasmática de eletrólitos do paciente. Por isso, deve-se ressaltar que apesar da baixa osmolaridade das soluções eletrolíticas enterais utilizadas no presente ensaio, principalmente na HipoMalt $(181 \mathrm{mOsm} / \mathrm{L})$, os animais não desenvolveram hiponatremia, o que é um bom indício. Enfatizando-se que nos casos de pacientes com hiponatremia, deve ser feito o monitoramento do sódio durante a hidratação, principalmente se esta for administrada por tempo prolongado. Diminuição significativa no valor do sódio plasmático de equinos normonatrêmicos em treinamento foi registrado por Sosa León et al. (1995b) e Marlin et al. (1998) após a hidratação enteral. Contudo, deve-se enfatizar que o decréscimo registrado no valor do sódio plasmático ocorreu nos animais que receberam água ao invés de solução eletrolítica enteral.
Quadro 2. Valores médios e desvio padrão de sódio ( $\left.\mathrm{mEq} \mathrm{L}^{-1}\right)$, potássio $\left(\mathrm{mEq} \mathrm{L}^{-1}\right)$, cloreto $\left(\mathrm{mEq} \mathrm{L}^{-1}\right)$, DIF $\left(\mathrm{mEq} \mathrm{L}^{-1}\right)$, cálcio iônico $\left(\mathrm{mMol} \mathrm{L} \mathrm{L}^{-1}\right)$ e magnésio (mg dL $\mathrm{m}^{-1}$ ) em equinos tratados com soluções eletrolíticas enterais contendo dextrose (SEDext), maltodextrina (SEMalt) ou propionato de cálcio (SEProp)

\begin{tabular}{|c|c|c|c|c|}
\hline Tratamentos & T0 & T6 & $\mathrm{T} 12$ & $\mathrm{~T} 24$ \\
\hline \multicolumn{5}{|c|}{ Sódio } \\
\hline SEDext & $141,67 \pm 4,03^{\mathrm{Aa}}$ & $143,67 \pm 4,46^{\mathrm{Aa}}$ & $143,33 \pm 3,39^{\text {Аа }}$ & $136,83 \pm 6,91^{\text {Aa }}$ \\
\hline SEMalt & $142 \pm 3,16^{\mathrm{Aa}}$ & $142 \pm 2,45^{\text {Аa }}$ & $139,83 \pm 0,75^{\text {Aab }}$ & $136,67 \pm 2,66^{\mathrm{Ab}}$ \\
\hline SEProp & $141,17 \pm 1,83^{\text {Aab }}$ & $142,83 \pm 2,4^{\mathrm{Aa}}$ & $142,67 \pm 3,5^{\text {Aab }}$ & $138,67 \pm 2,06^{\mathrm{Ab}}$ \\
\hline \multicolumn{5}{|c|}{ Potássio } \\
\hline SEDext & $4,72 \pm 0,78^{\text {Aab }}$ & $4,03 \pm 0,29^{\mathrm{Ab}}$ & $4,97 \pm 0,81^{\text {Aab }}$ & $5,37 \pm 0,22^{\mathrm{Aa}}$ \\
\hline SEMalt & $4,62 \pm 0,4 \mathrm{~A}^{\mathrm{ab}}$ & $4,15 \pm 0,23^{\text {Aab }}$ & $4,02 \pm 0,32^{\mathrm{Ab}}$ & $4,75 \pm 0,33^{\mathrm{Aa}}$ \\
\hline SEProp & $4,87 \pm 0,73^{\mathrm{Aa}}$ & $3,98 \pm 0,31^{\mathrm{Ab}}$ & $3,92 \pm 0,29^{\mathrm{Ab}}$ & $4,38 \pm 0,56^{\mathrm{Aa}}$ \\
\hline \multicolumn{5}{|c|}{ Cloreto } \\
\hline SEDext & $90,33 \pm 6,05^{\text {Аа }}$ & $98,5 \pm 4,50$ Аа & $97,00 \pm 7,92^{\text {Аа }}$ & $95,33 \pm 10,25^{\mathrm{Aa}}$ \\
\hline SEMalt & $93,83 \pm 6,67 \mathrm{Aa}$ & $92,66 \pm 12,67$ Аа & $93,50 \pm 12,52^{\mathrm{Aa}}$ & $95,5 \pm 12,66^{\mathrm{Aa}}$ \\
\hline SEProp & $95,83 \pm 6,43^{\mathrm{Aa}}$ & $96,17 \pm 8,49$ & $97,17 \pm 11,84$ & $89,83 \pm 8,66^{\mathrm{Aa}}$ \\
\hline \multicolumn{5}{|c|}{ DIF } \\
\hline SEDext & $53,47 \pm 7,59 \mathrm{Aa}$ & $48,46 \pm 6,88^{\mathrm{Aa}}$ & $51,36 \pm 10,43^{\mathrm{Aa}}$ & $47,87 \pm$ \\
\hline SEMalt & $51,22 \pm 7,91^{\mathrm{Aa}}$ & $53,98 \pm 13,42^{\text {Аа }}$ & $50,75 \pm 11,94$ & $46,92 \pm 11,28$ \\
\hline SEProp & $49,22 \pm 6,97$ Аа & $49,29 \pm 9,85^{\text {Аа }}$ & $47,39 \pm 11,51^{\mathrm{Aa}}$ & $51,06 \pm 8,96^{\mathrm{Aa}}$ \\
\hline \multicolumn{5}{|c|}{ Cálcio iônico } \\
\hline SEDext & $1,19 \pm 0,17^{\text {Аa }}$ & $1,13 \pm 0,11^{\mathrm{Ba}}$ & $1,15 \pm 0,09^{\mathrm{Aa}}$ & $1,3 \pm 0,09 \mathrm{Aa}$ \\
\hline SEMalt & $1,14 \pm 0,1^{\mathrm{Aa}}$ & $1,14 \pm 0,08^{\mathrm{ABa}}$ & $1,15 \pm 0,09^{\mathrm{Aa}}$ & $1,31 \pm 0,18 \mathrm{Aa}$ \\
\hline SEProp & $1,10 \pm 0,12^{\mathrm{Ab}}$ & $1,28 \pm 0,11^{\mathrm{Aab}}$ & $1,18 \pm 0,24^{\mathrm{Aab}}$ & $1,38 \pm 0,04 \mathrm{Aa}$ \\
\hline \multicolumn{5}{|c|}{ Magnésio } \\
\hline SEDext & $2,35 \pm 0,39^{\text {Aab }}$ & $1,98 \pm 0,16^{\mathrm{Aab}}$ & $1,95 \pm 0,43^{\mathrm{Ab}}$ & $2,57 \pm \pm 0,43^{\mathrm{Aa}}$ \\
\hline SEMalt & $2,37 \pm 0,44^{\text {Aab }}$ & $2,37 \pm 0,44^{\text {Aab }}$ & $1,93 \pm 0,21^{\mathrm{Ab}}$ & $2,77 \pm 0,24^{\mathrm{Aa}}$ \\
\hline SEProp & $2,73 \pm 0,21^{\mathrm{Aa}}$ & $1,97 \pm 0,2^{\mathrm{Ab}}$ & $2,25 \pm 0,25^{\mathrm{Ab}}$ & $2,83 \pm 0,24^{\mathrm{Aa}}$ \\
\hline
\end{tabular}

DIF = diferença de íons fortes. Análise de variância (medidas repetidas). As médias na mesma coluna, seguidas por letras maiúsculas diferentes, e as médias na mesma linha, seguidas por letras minúsculas diferentes, diferem pelo teste de Tukey $(p>0,05)$. T0h (imediatamente antes do início da hidratação), T6h (seis horas de hidratação), T12h (12 horas de hidratação) e T24h (12 horas após o término da hidratação).

As concentrações de potássio sérico não apresentaram diferença entre tratamentos ( $p>0,05)$, entretanto foi observada diferença em todos os tratamentos ao longo da fase experimental $(p<0,05)$. Houve decréscimo nos valores do potássio no T6 e T12 no tratamento IsoProp; no tratamento HipoDext, foi verificado diminuição no T6 em relação ao T24 e no HipoMalt o teor de potássio no T12 foi menor que no T24 (Quadro 2). Essa discreta redução nos valores médios de potássio que ocorreu nos animais de todos os tratamentos ao longo da fase experimental (T6 e T12), foi provavelmente ocasionada pela composição da solução eletrolítica associada à expansão do volume plasmático decorrente da hidratação. Apesar da diminuição, os valores do potássio mantiveram-se na faixa de referência (Kaneko et al. 2008) indicando que a quantidade de $\mathrm{KCl}, 0,5 \mathrm{~g}$ por litro de solução pode ser utilizada. Contudo, a dose de potássio pode atingir até $1 \mathrm{~g}$ por litro de solução eletrolítica (Ribeiro Filho et al. 2012). Esse valor (1 $\left.\mathrm{g} \mathrm{L}^{-1}\right)$ deve ser considerado nos casos de hipocalemia que pode ocorrer em pacientes com diarreia (Smith 2009). Discreta diminuição nos valores do potássio sérico também foi registrada por Avanza et al. (2009), após administração de solução eletrolítica enteral contendo $0,37 \mathrm{~g}$ de $\mathrm{KCl}$ por litro de solução, mas assim como a encontrada no presente ensaio, as concentrações do potássio se mantiveram na faixa de normalidade para a espécie equina. 
Não foi observada diferença nos valores médios do cloreto $(p>0,05)$ entre os tratamentos e nos tratamentos ao longo do tempo (Quadro 2). Esses resultados revelam que a quantidade de cloreto utilizada nas soluções eletrolíticas era análoga ao cloreto sanguíneo. Esse achado é importante, pois o excesso ou déficit desse íon no sangue predispõe o aparecimento de alterações no equilíbrio ácido base, acidose e alcalose metabólicas, respectivamente (Constable 2003; DeMorais \& Biondo 2012). Segundo Stewart (1983) somente a $\mathrm{pC}_{2}$, DIF e proteínas $\left(\mathrm{A}_{\mathrm{tot}}\right)$ podem determinar alteração no equilíbrio ácido base. Quando se observa os valores da DIF obtidos nos animais do presente estudo, percebe-se que não houve alteração $(p>0,05)$ entre tratamentos e nos tratamentos ao longo da fase experimental (Quadro 2), confirmando que o cloreto contido na solução eletrolítica não produziu modificação na sua concentração sérica, consequentemente não predispôs alteração no equilíbrio ácido base.

Ausência na alteração do cloreto sérico foi também registrada por Lopes et al. (2002), os quais usaram solução eletrolítica enteral em equinos contendo valores de $\mathrm{NaCl}$ semelhantes aos do presente ensaio. Por sua vez, Alves et al. (2005) relataram discreto aumento no valor do cloreto sérico ao utilizar soluções eletrolíticas enterais contendo quantidades maiores de $\mathrm{NaCl}$ e $\mathrm{KCl}$ quando comparadas as do presente estudo. Deve-se ressaltar que soluções eletrolíticas com quantidades maiores de cloreto são necessárias nos pacientes que apresentam alcalose metabólica ocasionada por hipocloremia, daí essas soluções serem denominadas de soluções acidificantes. Como as soluções testadas na presente pesquisa não apresentaram efeito alcalinizante, tampouco acidificante podem ser classificadas como neutras.

Como expressa no Quadro 2, os valores médios do cálcio iônico (iCa) no tratamento IsoProp diferiu do HipoDext no tempo $6 \mathrm{~h}(\mathrm{p}<0,05)$, ou seja, os valores do iCa no IsoProp foram superiores ao HipoDext. Além disso, no tratamento IsoProp foi constatado discreto aumento do iCa no T6h e $\mathrm{T} 12 \mathrm{~h}$, atingindo os maiores índices no T24h $(\mathrm{p}<0,05)$. Esse achado foi decorrente da composição da solução eletrolítica, o IsoProp continha maior concentração de cálcio que os demais tratamentos. Por sua vez, Avanza et al. (2009) observaram diminuição no cálcio sérico de equinos que receberam solução eletrolítica contendo maltodextrina por via enteral. Lopes et al. (2002) também relataram diminuição nos valores do cálcio total em equinos que receberam solução eletrolítica por via enteral, salientando-se que as soluções eletrolíticas enterais utilizadas pelos referidos autores não incluía cálcio como constituintes.

Não foram observadas alterações entre valores médios do magnésio nos tratamentos, mas houve diferença nos tratamentos ao longo da fase experimental $(p<0,05)$. No final da fase de hidratação (T12), foi observada diminuição do Mg em todos os tratamentos (Quadro 2). Possivelmente esse achado deveu-se a hemodiluição ocasionada pela expansão do volume plasmático decorrente da hidratação enteral como relatou Alves et al. (2005) e Avanza et al. (2009), sinalizando que a quantidade de magnésio das soluções eletrolíticas pode ser aumentada. Deve-se ressal- tar que, é prudente aumentar a quantidade de magnésio à solução eletrolítica nos casos em que o animal apresente hipomagnesemia, principalmente nos casos de cólica por estrangulamento, pois como citou Garcia-Lopez (2001) a hipomagnesemia é um achado comum nesses pacientes.

Durante a fase experimental houve diferença entre valores médios das proteínas plasmáticas totais nos tratamentos $(\mathrm{p}<0,05)$. No T6h o IsoProp apresentou valores superiores ao HipoDext e ao HipoMalt, enquanto no T12h o IsoProp foi superior ao HipoMalt (Quadro 3). Esses resultados sinalizam que as soluções enterais contendo dextrose e maltodextrina foram mais eficientes na expansão do volume plasmático, sendo essa ação ocasionada pela baixa osmolaridade das referidas soluções, $228 \mathrm{mOsm} \mathrm{L}^{-1} \mathrm{e}$ $181 \mathrm{mOsm} \mathrm{L}^{-1}$, respectivamente. Estudos experimentais demonstraram (Hunt et al. 1992, Rautanen et al. 1993, 1994, Nishinaka et al. 2004) que soluções hipotônicas proporcionam melhor absorção de água do que as isotônicas, confirmando os resultados da presente pesquisa. Os principais benefícios das soluções eletrolíticas com baixa osmolaridade são: reduz o risco de hipernatremia, aumenta a absorção de água e reduz a produção fecal (Ribeiro Filho 2011), o que as torna uma boa opção nos pacientes com diarreia.

A administração de soluções eletrolíticas por via enteral não ocasionou alteração nos valores de creatinina entre

Quadro 3. Valores de proteínas plasmáticas totais $\left(\mathrm{g} \mathrm{dL}^{-1}\right)$, ureia $\left(\mathrm{mg} \mathrm{dL}^{-1}\right)$, creatinina $\left(\mathrm{mg} \mathrm{dL}^{-1}\right)$, glicose $\left(\mathrm{mg} \mathrm{dL}^{-1}\right)$, lactato ( $\left.\mathrm{mMol} \mathrm{L}^{-1}\right)$ e osmolaridade $\left(\mathrm{mMol} \mathrm{L}^{-1}\right)$ em equinos tratados com soluções eletrolíticas enterais contendo dextrose (SEDext), maltodextrina (SEMalt) ou propionato de cálcio (SEProp)

\begin{tabular}{|c|c|c|c|c|}
\hline Tratament & T0 & T6 & T12 & $\mathrm{T} 24$ \\
\hline \multicolumn{5}{|c|}{ Proteínas plasmáticas totais } \\
\hline SEDext & $7,07 \pm 0,57^{\mathrm{Aa}}$ & $7,02 \pm 0,43^{\mathrm{Ba}}$ & $7,12 \pm 0,36^{\mathrm{ABa}}$ & $7,08 \pm 0,53^{\text {Aa }}$ \\
\hline SEMalt & $7,13 \pm 0,35^{A a}$ & $6,85 \pm C$ & 6,90 & $7,47 \pm$ \\
\hline SEProp & $7,60 \pm 0,48^{\text {Aa }}$ & $\begin{array}{c}7,85 \pm 0,51^{\text {Aa }} \\
\text { Ureia }\end{array}$ & $7,68=$ & 7,93 \\
\hline SEDext & $35 \pm 3,46$ & $29 \pm 3$ & $26,5 \pm 3,78^{\mathrm{Ab}}$ & $26,5 \pm 3,5^{\mathrm{Ab}}$ \\
\hline SEMalt & $39,33 \pm 6,7$ & $33,17 \pm 7$ & $24,83 \pm$ & $26,17 \pm 4,12^{\mathrm{Ab}}$ \\
\hline SEProp & $37,5 \pm 5,43^{\mathrm{Aa}}$ & $\begin{array}{r}28,67 \pm 2 \\
\text { Creatin }\end{array}$ & $25 \pm 4.94^{\mathrm{Ab}}$ & $27,5 \pm 3,88^{\mathrm{Ab}}$ \\
\hline SEDext & $0,75 \pm 0,10^{\mathrm{Aa}}$ & $0,68 \pm$ & 0,73 & 0,70 \\
\hline SEMalt & $0,77 \pm$ & $0,73 \pm$ & 0,7 & 0,70 \\
\hline SEProp & $0,77 \pm 0,12^{\text {Aa }}$ & $0,70 \pm 0,00^{A}$ & 0,72 & 0,67 \\
\hline \multicolumn{4}{|c|}{ Glicose } & \\
\hline $\mathrm{SH}$ & 79,67 & 102,50 & $10^{7}$ & 86, \\
\hline SEProp & $93,50 \pm$ & $\begin{array}{c}83,50 \pm 11,08^{\mathrm{Ba}} \\
\text { Lactato }\end{array}$ & Ba & 96,67 \\
\hline SEDext & $0,90 \pm 0,28^{\text {Аа }}$ & $0,73 \pm 0,28^{\mathrm{Ba}}$ & $0,60 \pm 0,18^{\mathrm{Ba}}$ & $0,96 \pm 0,29^{\text {Aa }}$ \\
\hline SEMalt & $0,89 \pm 0,17^{\text {Аа }}$ & $0,83 \pm 0,25^{\text {Ba }}$ & $0,93 \pm 0,38^{\mathrm{ABa}}$ & $0,90 \pm 0,13^{\text {Aa }}$ \\
\hline SEProp & $0,97 \pm 0,11^{\text {Aa }}$ & $1,35 \pm 0,15^{\text {Аa }}$ & $1,36 \pm 0,44^{\mathrm{Aa}}$ & $1,15 \pm 0,17^{\text {Аа }}$ \\
\hline \multicolumn{5}{|c|}{ Osmolaridade sérica } \\
\hline SEDext & $280,00 \pm 4,69^{\text {Аа }}$ & $277,50 \pm 3,02^{\text {Аа }}$ & $276,00 \pm 7,13^{\text {Aa }}$ & $277,83 \pm 2,64^{\mathrm{Aa}}$ \\
\hline SEMalt & $280,50 \pm 4,55^{\text {Аа }}$ & $278,00 \pm 4,65^{\mathrm{Aa}}$ & $280,33 \pm 2,58^{\text {Аа }}$ & $276,00 \pm 2,97^{\text {Aa }}$ \\
\hline SEProp & $281,00 \pm 1,55^{\text {Аа }}$ & $281,67 \pm 7,37^{\text {Аа }}$ & $279,17 \pm 5,71^{\mathrm{Aa}}$ & $277,67 \pm 6,4$ \\
\hline
\end{tabular}

Análise de variância (medidas repetidas). As médias na mesma coluna, seguidas por letras maiúsculas diferentes, e as médias na mesma linha, seguidas por letras minúsculas diferentes, diferem pelo teste de Tukey ( $p>0,05$ ). T0h (imediatamente antes do início da hidratação), T6h (seis horas de hidratação), T12h (12 horas de hidratação) e T24h (12 horas após o término da hidratação). 
tratamentos, tampouco nos tratamentos ao longo da fase experimental $(\mathrm{p}>0,05)$. Porém, observou-se decréscimo $(\mathrm{p}<0,05)$ na concentração de uréia, em todos os tratamentos, durante o período de hidratação (T6h e T12h), demonstrando que houve expansão da volemia e consequente aumento da perfusão renal, determinando sua maior excreção por via renal (Quadro 3), confirmando os resultados obtidos por Alves et al. (2005).

Durante a fase de hidratação, a concentração de glicose apresentou diferença entre os tratamentos (Quadro 3). No T6h, os animais dos tratamentos HipoDext e HipoMalt apresentaram maiores valores de glicose plasmática $(\mathrm{p}<0,05)$ quando comparados aos do IsoProp, enquanto no T12h os animais que receberam a solução eletrolítica com maltodextrina (HipoMalt) exibiram maior índice de glicose quando comparados aos da solução com propionato de cálcio (IsoProp). Resultados similares ao presente ensaio foram registrados por Pessin (2012) ao comparar o efeito de soluções eletrolíticas enterais com maltodextrina, dextrose ou sacarose sobre a glicose plasmática. A eficiência da maltodextrina também foi citada por Nunes et al. (2012) ao utilizar dietas com diferentes quantidades desse carboidrato em equinos. Os referidos pesquisadores detectaram aumento na taxa glicêmica dos animais em comparação aos que receberam somente amido na dieta, além disso, o acréscimo nos valores da glicose sanguínea detectado nos animais alimentados com maltodextrina persistiu por mais tempo. Os resultados citados acima reforçam os obtidos na presente pesquisa.

Apesar do aumento nos valores da glicose em todos os tratamentos durante a fase de hidratação dos animais (T6h e T12h) não ter sido significativo $(p>0,05)$ e não ter ultrapassado o faixa de referência (75-125mg/dL), o HipoDext aumentou em 21,73\% a taxa glicêmica, enquanto o HipoMalt o acréscimo foi de 34,49\%. Por sua vez, no IsoProp esse acréscimo não ocorreu (Quadro 3), o que foi um resultado inesperado, pois esperava-se que o propionato de cálcio também tivesse efeito positivo sobre a taxa glicêmica, como ocorre em bovinos (Rufino et al. 1997), entretanto isso não aconteceu.

Baseado nos resultados do presente ensaio, a maltodextrina e a dextrose são as fontes de energia mais eficazes para uso enteral em equinos. Ambas tem um alto índice glicêmico, porém a maltodextrina a vantagem de manter a glicose sanguínea elevada por mais tempo (Nunes et al. (2012). Como o uso de soluções eletrolíticas enterais em equinos tem aumentado, tanto nos enfermos desidratados, assim como nos que desenvolvem desidratação após atividades físicas, a utilização dessas soluções acrescidas de uma fonte de energia muitas vezes é essencial para manter o balaço energético do animal. O seu uso é imperativo em animais que apresentem uma doença base que ocasione hipoglicemia, assim na recomposição do glicogênio muscular após o exercício, pois como citou Waller \& Lindinger (2010) a desidratação pode afetar a sua síntese.

Durante o período de hidratação (T6h e T12h), o lactato plasmático apresentou diferença entre os tratamentos $(\mathrm{p}<0,05)$. 0 grupo IsoProp apresentou maiores valores de lactato que os demais tratamentos, enquanto nos tratamen- tos ao longo do tempo não se detectou diferença $(p>0,05)$. Avaliando-se os valores do lactato, percebe-se também que apesar de não haver diferença $(p>0,05)$, os valores do lactato nos animais do tratamento IsoProp durante a fase experimental (T6h e T12h) aumentaram em 40,2\%, enquanto na maltodextrina (HipoMalt) foi 3,56\% e a dextrose (HipoDext) decresceu 33,3\%.

A provável causa para o aumento do lactato plasmático nos animais que receberam o tratamento IsoProp foi a ação da microbiota intestinal sobre o propionato de cálcio, ou seja, o propionato pode ter desencadeado aumento no processo fermentativo que por sua vez aumentou os valores do lactato. Cruz (2008) administrando solução eletrolítica enteral contendo propionato de cálcio em equinos também relatou o aumento do lactato plasmático após 12 horas de hidratação. Esses resultados indicaram que o propionato de cálcio não é uma boa opção como fonte de energia para equinos, pois além de não apresentar efeito positivo sobre a taxa glicêmica, ocasiona aumento do lactato plasmático, distensão abdominal bilateral e diarreia (Farias 2010).

No Quadro 3 pode-se constatar a ausência de diferença dos valores médios da osmolaridade sérica nos tratamentos e nos tratamentos durante todo o período experimental ( $p>0,05)$. Independente da osmolaridade das soluções eletrolíticas utilizadas no presente ensaio, duas hipotônicas e uma isotônica, a osmolaridade sérica dos animais se manteve próxima da faixa de referência (Kaneko et al. 2008).

A osmolaridade das soluções eletrolíticas enterais ainda é motivo de controversa entre pesquisadores. Nos animais, a maioria dos estudos foi realizada com soluções eletrolíticas enterais isotônicas (Lopes et al. 2002, Avanza et al. 2009, Gomes et al. 2012, Ribeiro Filho et al. 2012), por isso ainda sabe-se pouco sobre os efeitos das soluções hipotônicas nos animais, o que demonstra a importância do presente ensaio. 0 grande questionamento a respeito do uso das soluções enterais hipotônicas destaca-se o seu potencial de induzir hiponatremia no paciente, principalmente naquele que já apresenta valores séricos de sódio diminuídos. Apesar do presente ensaio ter sido realizado com animais osmolaridade na faixa de normalidade (280$300 \mathrm{mmol} / \mathrm{L}$ ), acredita-se a solução eletrolítica acrescida de maltodextrina (HipoMalt), apesar da sua baixa osmolaridade, pode ser utilizada. Contudo, em animais com hiponatremia intensa, sódio sérico menor que $120 \mathrm{mmol} / \mathrm{L}$, ela deve ser utilizada cautela associada ao monitoramento do sódio sérico do paciente durante o período de hidratação. Ressaltando-se que até o momento, em humanos, não foi relatado na literatura o aparecimento de hiponetremia decorrente do uso de soluções eletrolíticas enterais, o que levou a Organização Mundial de Saúde, após anos de estudo, a recomendar o uso de uma solução oral hipotônica para crianças desde 2005.

\section{CONCLUSÕES}

Soluções eletrolíticas hipotônicas contendo dextrose (HipoDext) e maltodextrina (HipoMalt) aumentam a taxa glicêmica e não ocasionam desequilíbrio eletrolítico, o que as torna uma opção para hidratação enteral em equinos. 
A solução eletrolítica com propionato de cálcio (IsoProp) não ocasiona efeito positivo sobre a glicemia.

A utilização dos tratamentos HipoDext HipoMalt na presente pesquisa gerou informações importantes para o uso de soluções eletrolíticas enterais em equinos.

\section{REFERÊNCIAS}

Alves G.E.S., Ribeiro Filho J.D., Oliveira H.P. \& Abreu J.M.G. 2005. Tratamento da compactação experimental do cólon maior em equinos: resultados de laboratório e exames bioquímicos. Arq. Bras. Med. Vet. Zootec. 57:281-287.

Avanza M.F.B., Ribeiro Filho J.D., Lopes M.A.F., Ignácio F.S., Carvalho T.A. \& Guimarães J.D. 2009. Hidratação enteral em equinos - solução eletrolítica associada ou não à glicose, à maltodextrina e ao sulfato de magnésio: resultados de laboratório. Ciencia Rural 39:1126-1133.

Constable P.D. 2003. Hypercloremic acidosis: the classic example of Strong ion acidosis. Anesth. Analg. 96:919-922.

Cruz A.L. 2008. Avaliação dos efeitos de soluções eletrolíticas enterais sobre parâmetros clínicos e bioquímicos de equinos desidratados experimentalmente. Monografia de Especialização em Clínica e Cirurgia de Grandes Animais, Departamento de Medicina Veterinária, Universidade Federal de Viçosa, Viçosa, MG. 37p.

DeMorais H.A. \& Biondo A.W. 2012. Disorders of chloride: hyperchloremia and hypochloremia, p.80-91. In: DiBartolla S.P. (Ed.), Fluid, Electrolyte and Acid-Base Disorders in Small Animal Practice. $4^{\text {th }}$ ed. Elsevier, St Louis.

Donner A.C. 2013. Efeitos da ingestão ad libitum de repositor hidroeletrolítico e energético em equinos submetidos ao treinamento de marcha. Dissertação de Mestrado em Medicina Veterinária, Departamento de Veterinária, Universidade Federal de Viçosa, Viçosa, MG. 60p.

Farias S.K. 2010. Efeitos de soluções eletrolíticas associadas ou não à dextrose, maltodextrina e propionato de cálcio administradas por via enteral sobre parâmetros clínicos e laboratoriais de equinos. Dissertação de Mestrado em Medicina Veterinária, Departamento de Veterinária, Universidade Federal de Viçosa, Viçosa, MG. 73p.

Farias S.K., Ribeiro Filho J.D., Donner A.C., Dantas W.M.F. \& Gomes C.L.N. 2011. Hemogasometria e ânion gap em equinos tratados com soluções eletrolíticas enterais contendo diferentes fontes de energia. Ciência $\mathrm{Ru}-$ ral 41:1587-1592.

Garcia-Lopez J.M. 2001. Prevalence e prognostic importance of hypomagnesemia and hypocalcemia in horses that have colic. Am. J. Vet. Res. 62:7-12.

Gomes C.L.N., Ribeiro Filho J.D., Guimarães J.D. \& Menezes R.M. 2012. Efeitos do polietilenoglicol 3350 e de soluções acidificantes e alcalinizantes sobre o perfil bioquímico de éguas hígidas. Arq. Bras. Med. Vet. Zootec. 64:817-826.

Hunt J.B., Thillainayagam A.V., Salim A.F., Carnaby S., Elliott E.J. \& Farthing M.J. 1992. Water and solute absorption from a new hypotonic oral rehydration solution: evaluation in human and animal perfusion models. Gut 33:1652-1659.

Kaneko J.J., Harvey J.W. \& Bruss M.L. 2008. Clinical Biochemistry of Domestic Animals. Academic, San Diego. 932p.

Lindinger M.I. \& Ecker G.L. 2013. Gastric emptying, intestinal absorption of electrolytes and exercise performance in electrolyte-supplemented horses. Exp. Physiol. 98:193-206.

Lopes M.A.F., Walker B.L., White N.A. \& Ward D.L. 2002. Treatments to promote colonic hydration: enteral fluid therapy versus intravenous therapy e magnesium sulphate. Equine Vet. J. 34:505-509.

Marlin D.J., Scott C.M., Mills P.C., Louwes H. \& Vaarten J. 1998. Effects of administration of water versus an isotonic oral rehydration solution at rest and changes during exercise and recovery. Vet. J. 155:69-78.
McClure J.T. 2001. Oral fluid therapy for treatment of neonatal diarrhea in calves. Vet. J. 162:87-89.

Nishinaka D., Kishino F. \& Matsuura A. 2004. Water and electrolyte absorption from hypotonic oral rehydration solution in rat small intestine and colon. Pediatr. Int. 46:315-321.

Nunes Gil P.C., Gandra J.R., Taran F.M.P., Gonzaga I.V.F. \& Gobesso A.A.O. 2012. Infleunce of high level maltodextrin in horse diet. Livest. Sci. 147:66-71.

Pessin A.E. 2013. Efeitos de soluções eletrolíticas hipotônicas associadas à sacarose, dextrose ou maltodextrina administradas por via enteral em equinos. Dissertação de Mestrado em Medicina Veterinária, Departamento de Veterinária, Universidade Federal de Viçosa, Viçosa, MG. 79p.

Pessin A.E., Ribeiro Filho J.D., Donner A.C., Oliveira D.P., Lima A.P. \& Fonseca B.P.A. 2013. Soluções eletrolíticas enterais hipotônicas em equinos: efeitos de fontes de energia sobre determinados indicadores do equilíbrio ácido base. Ciência Rural 43:2240-2246.

Rainger J.E. \& Dart A.J. 2006. Enteral fluid therapy in large animals. Aust. Vet. J. 84:447-451.

Rautanen T., El-Radhi S. \& Vesikari T. 1993. Clinical experience with a hypotonic oral rehydration solution in acute diarrhoea. Acta Paediatr. 82:52-54.

Rautanen T., Salo E., Verkasalo M. \& Vesikari T. 1994. Randomised double blind trial of hypotonic oral rehydration solutions with and without citrate. Arch. Dis. Child. 70:44-46.

Ribeiro Filho J.D. 2011. Soluções eletrolíticas com baixa osmolaridade são eficazes na hidratação enteral ? Vet. Zootec. 18:47-50.

Ribeiro Filho J.D., Abreu J.M.G., Alves G.E.S. \& Dantas W.M.F. 2007. Hemogasometria em equinos com compactação experimental do cólon maior tratados com sene, fluidoterapia enteral e parenteral. Ciência Rural 37:755-761.

Ribeiro Filho J.D., Gimenes A.M., Fonseca E.F., Dantas W.M.F. \& Oliveira T.T. 2011. Hidratação enteral em bovinos: avaliação de soluções eletrolíticas isotônicas administradas por sonda nasogástrica em fluxo contínuo. Ciência Rural 41:285-290.

Ribeiro Filho J.D., Alves G.E.S. \& Dantas W.M.F. 2012. Tratamentos da compactação experimental do cólon maior de equinos com hidratação enteral, intravenosa e sene (Cassia augustifolia Vahl). Revta Ceres 59:32-38.

Rufino L., Krehbiel C. \& Kloppenburg P. 1997. Effects of nutrocal fed in a peripartum period on serum nutrient profiles and milk production in Holstein cows. Technical Information, Kemin Industries, Des Moines, p.1-8

SAS 2000. User's guide. Statistical Analysis System. Version 8.1, SAS Institute. Inc., Cary.

Sosa León L.A., Davie A.J., Hodgson D.R., Evans D.L. \& Rose R.J. 1995a. Effects of oral-fluid on cardiorespiratory and metabolic responses to prolonged exercise. Equine Vet. J. 27:274-278.

Sosa León L.A., Davie A.J., Hodgson D.R. \& Rose R.J. 1995b. The effects of tonicity, glucose concentration and temperature of an oral rehydration solution on its absortion and elimination. Equine Vet. J. 20:140-146.

Smith G.W. 2009. Treatment of calf diarrhea: oral fluid therapy. Vet. Clin. North Am., Food Anim. Pract. 25:55-72.

Speirs V.C. 1997. Exame Clínico de Equinos. Artmed, Porto Alegre. 286p.

Stewart P.A. 1983. Modern quantitative acid-base chemistry. Can. J. Physiol. Pharmacol. 61:1444-1461.

Teixeira-Neto A.R., Ferraz G.C., Mataqueiro M.I., Lacerda-Neto J.C. \& Queiroz-Neto A. 2004. Reposição eletrolítica sobre variáveis fisiológicas de cavalos em provas de enduro de 30 e 60 km. Ciência Rural 34:15051511.

Waller A.P. \& Lindiger M.I. 2010. Nutritional aspects of post exercise skeletal muscle glycogen synthesis in horses: a comparative review. Equine Vet. J. 42:274-281. 